\title{
Axial Anomalies and Index Theorems on Open Spaces *
}

\section{Constantine Callias}

Center for Theoretical Physics, Laboratory for Nuclear Science and Department of Physics, Massachusetts Institute of Technology, Cambridge, Massachusetts 02139, USA

\begin{abstract}
Using an approach inspired by the theory of the anomalous divergence of the axial vector current, we derive trace formulas for the resolvents of Dirac operators on open spaces of odd dimension. These formulas readily yield index theorems for these operators. As applications we determine the index of the Dirac operator for a particle of arbitrary isospin in the background field of a static system of SU(2) monopoles; and we find formulas in essentially closed form for certain determinants involving these operators.
\end{abstract}

\section{Introduction}

It is the purpose of this paper to give an elementary analytical proof of an index theorem for Dirac operators on open Euclidean spaces arising in Minkowski space-time Yang-Mills theories. We use basically an extension to open spaces of the index formulas in terms of traces of the propagation operators for the heat equation.

The index theorem has so far been applied to physical systems that can be stereographically projected onto a compact manifold [1]. Thus for the case of pseudoparticle configurations in Euclidean (imaginary time) Yang-Mills theory in four dimensions, it has been used to determine the number of zero eigenvalue modes of the Dirac equation and the number of independent parameters of selfdual or anti-dual solutions [2].

The possibility of compactifying the problem is the basic feature of these systems that makes the Atiyah-Singer [3] index theorem applicable. Compactification is actually necessary on a more fundamental level in these cases: to make the problem well-defined; precisely the operators involved are not Fredholm on the open space. The even dimensionality of the projected manifold guarantees that at least a priori the index problem can be non-trivial.

* This work is supported in part through funds provided by the U.S. Department of Energy (DOE) under contract EY-76-C-02-3069 
However, examples are known of Dirac equations in Minkowski space of an odd number of space-dimensions with non-degenerate static (time-independent) modes [4]. Writing such a Dirac equation in the form

$$
\left(\begin{array}{cc}
0 & L \\
L^{\dagger} & 0
\end{array}\right) \psi=i \partial_{i} \psi
$$

where $L$ is an elliptic operator on odd-dimensional Euclidean space, we see that $L$ has a nonvanishing index.

Unlike the case of the pseudoparticle configurations, the index problem for $L$ is well-defined on the open space, as we show in the next section. On the other hand, a compactification of the problem, which may be possible, will necessarily lead to an inequivalent index problem : the index of any elliptic differential operator on an odd dimensional compact manifold is known to be zero [3]. Thus the static modes in the type of situation described above must be explained by an index theorem on open Euclidean space.

We derive such an open space index theorem for Euclidean space of odd dimension. This theorem can also be proved more simply by using topological methods, as explained in the note by R. Bott and R. Seeley following this article. Our proof however only exemplifies the possibility of the use of the heat equation in open space index theory. The general idea is to use traces of the type [5]

$$
\operatorname{Tr}\left(e^{-t L^{\dagger} L}-e^{-t L L^{\dagger}}\right)
$$

or

$$
\operatorname{Tr}\left[\left(\frac{z}{L^{\dagger} L+z}\right)^{s}-\left(\frac{z}{L L^{\dagger}+z}\right)^{s}\right] .
$$

On a compact manifold, either of these traces gives the index for any value of $t$ or $z$ (for $s \gg 1$ ). On an open space we get the index by taking the limit $t \rightarrow \infty$ for the first one or $z \rightarrow 0$ for the second. Although the first trace is defined for a much wider class of problems, we use the second one, with $s=1$, which only happens to make sense due to the special form of the Dirac operator. The nice feature of this trace is its connection with the so-called anomalous divergence of the axial vector current [6]. A physicist will have no trouble recognizing this connection in the disguise of Proposition 1.

The class of Dirac operators that we study arises in Yang-Mills theories with both gauge and Higgs fields [4]. The most general Dirac equation appearing in this context is given by (1.1) below where $A_{i}(x)$ is related to the gauge field and $\Phi(x)$ to the Higgs field. We begin by giving the background on these equations. In Sect. II we then describe how the trace of the resolvent above can be computed and then used to derive index theorems for first order operators. In Sect. III we use these ideas to derive explicit trace formulas and the index theorem for our Dirac operators in odd dimension. Finally in Sect. IV we apply our results to systems of fermions and SU(2) monopole configurations. We conclude by discussing the relation of our results to the anomalous divergence of the axial vector current and to anomalous determinants. 


\section{The Problem - A Class of Fredholm Operators}

The index problems that we are concerned with in this paper arise from Dirac equations in Minkowski space-time of $n$ space and one time dimensions. Such equations are of the general form

$$
\left[i \alpha^{i} \partial_{i} \otimes 1_{m}+\alpha^{i} \otimes A_{i}(x)-\beta \otimes \Phi(x)\right] \psi(x, t)=-i \partial_{t} \psi(x, t) .
$$

Here $\psi(x, t)$ is a $2 p m$-component spinor. The $2 p \times 2 p$ Dirac matrices are given by

$$
\alpha^{i}=\left(\begin{array}{cc}
0 & \delta^{i} \\
\delta^{i} & 0
\end{array}\right) \quad \beta=i\left(\begin{array}{cc}
0 & -1_{p} \\
1_{p} & 0
\end{array}\right),
$$

where the $n p \times p$ matrices $\delta^{i}$ satisfy a Euclidean Dirac algebra:

$$
\delta^{i} \delta^{j}+\delta^{j} \delta^{i}=2 \delta^{i j} 1_{p}
$$

The tensor product in (1.1) is just the tensor product of the finite-dimensional matrices. Finally the coefficients $A_{i}(x), \Phi(x)$ are given by Hermitian $m \times m$ matrices.

They are assumed differentiable and bounded in $x$, and furthermore $\lim _{|x| \rightarrow \infty} A_{i}(x)=0$ and $\Phi(x)$ approaches a homogeneous function of order 0 as $|x| \rightarrow \infty$.

We can separate the time variable letting $\psi(x, t)=\psi(x) e^{i E t}$ and express (1.1) as an eigenvalue problem of the form:

$$
H \psi=\left(\begin{array}{cc}
0 & L \\
L^{\dagger} & 0
\end{array}\right)\left(\begin{array}{l}
\psi_{+} \\
\psi_{-}
\end{array}\right)=E\left(\begin{array}{l}
\psi_{+} \\
\psi_{-}
\end{array}\right),
$$

where $L$ is a first order $p m \times p m$ matrix differential operator on $\mathbb{R}^{n}$ :

$$
L=i \delta^{i} \partial_{i} \otimes 1_{m}+\delta^{i} \otimes A_{i}(x)+i 1_{p} \otimes \Phi(x) .
$$

$L^{\dagger}$ is the Hilbert space adjoint of $L$.

We are interested in the static ( $t$-independent) solutions of (1.1), i.e. the $E=0$ eigenspace of (1.2). For these solutions (1.2) implies

$$
\begin{aligned}
& L \psi_{-}=0 \\
& L^{\dagger} \psi_{+}=0 .
\end{aligned}
$$

Thus the dimension of the $E=0$ space is given by $k=k_{+}+k_{-}$, where $k_{+}=\operatorname{dim} \operatorname{Ker}\left(L^{\dagger}\right), k_{-}=\operatorname{dim} \operatorname{Ker}(L)$. We cannot in general determine $k$, but we can find a formula for

$$
\text { index }(L)=k_{-}-k_{+}=\operatorname{dim} \operatorname{Ker}(L)-\operatorname{dim} \operatorname{Ker}\left(L^{\dagger}\right)
$$

in terms of the behavior of the operator $L$ at $\infty$ in $\mathbb{R}^{n}$. This formula will determine

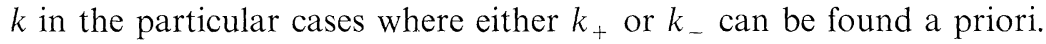

The operator $L$ is regarded as a linear transformation on the Hilbert space $\left[L^{2}\left(\mathbb{R}^{n}\right)\right]^{p m}$ and the adjoint is defined with respect to the inner product in this space. Now choose the domain of $L$ to be those functions in $\left[L^{2}\left(\mathbb{R}^{n}\right)\right]^{p m}$ that are also in $\left[H^{1}\left[\mathbb{R}^{n}\right)\right]^{p m}$, the first Sobolev space. $L$ is a closed operator on this domain and the above definition of the index agrees with the usual one for the bounded 
operator

$$
\tilde{L}:\left[H^{1}\left(\mathbb{R}^{n}\right)\right]^{p m} \rightarrow\left[L^{2}\left(\mathbb{R}^{n}\right)\right]^{p m}
$$

given by

$$
\text { index }(\tilde{L})=\operatorname{dim} \operatorname{Ker}(\tilde{L})-\operatorname{dim}(\operatorname{Ran} \tilde{L}) \text {. }
$$

To see this, observe simply that $\operatorname{Ker} \tilde{L}=\operatorname{Ker} L$ and $(\operatorname{Ran} \tilde{L})=\operatorname{Ker} L^{\dagger}$. For the first note that $\operatorname{Ker} \tilde{L} \subset \operatorname{Ker} L$, since $H^{1}\left(R^{n}\right) \subset L^{2}\left(R^{n}\right)$, and $\operatorname{Ker} L \subset \operatorname{Ker} \tilde{L}$, since $L \phi=0$ implies that $i \delta^{i} \partial_{i} \phi=B \phi$ where $B$ is bounded and this in turn implies that $\phi \in\left[H^{1}\left(\mathbb{R}^{n}\right)\right]^{p m}$, hence also in $\operatorname{Ker} \tilde{L}$. That $(\operatorname{Ran} \tilde{L})=\operatorname{Ker} L^{\dagger}$ follows from the definition of the adjoint.

By definition [7], $L$ is Fredholm if both $k_{-}$and $k_{+}$are finite and $L$ is closed. It follows that if $L$ is Fredholm, $\operatorname{Ran} L$ is closed. Now if $L$ is Fredholm, so are $L^{\dagger}$, $L L^{\dagger}$, and $L^{\dagger} L$. Since $L L^{\dagger}$ is self-adjoint, the fact that $\operatorname{Ran}\left(L L^{\dagger}\right)$ is closed implies that if 0 is in $\operatorname{Spec}\left(L L^{\dagger}\right)$ (the spectrum of $\left.L L^{\dagger}\right)$, it is an isolated eigenvalue. Similarly for $L^{\dagger} L$. Conversely, it is easy to see that if $L^{\dagger} L$ is Fredholm, so is $L$. We will use these facts below.

A simple criterion for self-adjoint operators to be Fredholm is the following. If $A$ and $B$ are self-adjoint operators such that $A \geqq B$ [which means that $(\phi, A \phi) \geqq(\phi, B \phi)$ for all $\phi]$ and $B$ is Fredholm then $A$ is Fredholm. This is an immediate consequence of the minimax principle [8].

A property of the index [7] which is extremely useful in the derivation of the index formula is its homotopy invariance. We only need in this paper the following restricted version of this property: If $t \rightarrow L(t)$ is a norm continuous map of the inverval $[0,1]$ into the space of Fredholm operators then index $(L(0))=$ index $(L(1))$.

A related invariance property [7] that we shall make use of below is under a perturbation that is compact relative to the original operator. By definition if $\mathscr{H}$ is a Hilbert space, $B: \mathscr{H} \rightarrow \mathscr{H}$ is compact relative to $L: D(L) \rightarrow \mathscr{H}, D(L) \subset \mathscr{H}$, if $B$ is compact as an operator: $D(L) \rightarrow \mathscr{H}$ where $D(L)$ is equipped with the norm $\|\cdot\|+\|L \cdot\|$.

It is therefore important to determine a general class of Fredholm operators as the setting for a given index problem. For our problem, we will restrict ourselves to a class no larger than that given by (1.3), with the conditions on $A_{i}(x), \Phi(x)$ preceding that equation. We need however precise conditions that tell us when an operator of the form (1.3) is Fredholm. These we state as a corollary to the following theorem of Seeley [9] on differential operators on $\mathbb{R}^{n}$.

Theorem 1. Let ${ }^{1} A=\sum_{|\alpha| \leqq m} a_{\alpha}(x)\left(i \frac{\partial}{\partial x}\right)^{\alpha}$ be a differential operator from the Sobolev space $\left[H^{k+m}\left(\mathbb{R}^{n}\right)\right]^{M}$ to $\left[H^{k}\left(\mathbb{R}^{n}\right)\right]^{M}$ where the $a_{\alpha}(x)$ are bounded and their derivatives are continuous and vanish at $\infty$. Then $A$ is Fredholm if there are constants $c$ and $C$ such that

$$
\left|\sum_{|\alpha|=m} a_{\alpha}(x) \xi^{\alpha}\right| \geqq c|\xi|^{m} \quad \text { all } \quad x \in \mathbb{R}^{n}
$$

(i.e. $A$ is uniformly elliptic) and

$$
\left|\sum_{|\alpha| \leqq m} a_{\alpha}(x) \xi^{\alpha}\right|
$$

We use multi-index notation: $\alpha=\left(\alpha_{1}, \ldots, \alpha_{n}\right),\left(\frac{\partial}{\partial x}\right)^{\alpha}=\frac{\partial^{\alpha_{1}}}{\partial x_{1}^{\alpha}} \ldots \frac{\partial^{\alpha_{n}}}{\partial x_{n}^{\alpha}}$. 
is bounded away from 0 for $|x| \geqq C$. Conversely, if $A$ is Fredholm from $\left[H^{m}\left(\mathbb{R}^{n}\right)\right]^{M}$ to $\left[L^{2}\left(\mathbb{R}^{n}\right)\right]^{M}$ then there exist such constants $c$ and $C$.

Since our deformation arguments on the index are restricted in the class of operators of the form (1.3), all we need in this paper is the sufficiency statement of this theorem for operators of the form (1.3). Although we state the following theorem as a corollary, we give an independent elementary proof, which is possible because of the very special form of the operators that we study.

Corollary. The operator $L$ defined by (1.3) is Fredholm if $|\Phi(x)| \geqq B$ for $|x| \geqq C$, where $B, C$ are positive constants. The index of $L$ is equal to the index of $\tilde{L}$ if $\tilde{L}$ is an elliptic operator such that

$$
\tilde{L}=i \delta^{i} \partial_{i} \otimes 1_{m}+i 1_{p} \otimes U(x)
$$

for $|x|>C$, where $U(x)$ is the Hermitian unitary matrix $|\Phi(x)|^{-1} \Phi(x)$, $|\Phi(x)|=\left[\Phi(x)^{\dagger} \Phi(x)\right]^{1 / 2}$.

Proof. Let

$$
L_{1}=i \delta^{i} \partial_{i} \otimes 1_{m}+i 1_{p} \otimes \Phi(x) .
$$

$L$ is Fredholm if and only if $L_{1}$ is Fredholm since the fact that the $A_{i}(x)$ are bounded and vanish at $\infty$ implies that the term $\delta^{i} \otimes A_{i}(x)$ in (1.3) is $L_{1}$-compact, by the Rellich lemma [10] (see above). Now notice that

$$
L_{1}^{\dagger} L_{1}=-\partial^{2} 1-\delta^{i} \otimes \Phi_{, i}(x)+1_{p} \otimes|\Phi(x)|^{2} .
$$

Since $\Phi(x)$ is $C^{\infty}$ and asymptotically homogeneous we have

$$
L_{1}^{\dagger} L_{1} \geqq-\partial^{2} \cdot 1-\frac{x}{|x|} \cdot 1+B \cdot 1
$$

where $B$ is such that $|\Phi(x)|^{2}>B$. The operator on the righthand side has a discrete spectrum of eigenvalues of finite multiplicity contained in $(-\infty, B)$, so that if $B>0$ it is Fredholm. Then so is $L_{1}^{\dagger} L_{1}$ and therefore $L_{1}$ as well.

The one parameter family of operators

$$
L(t)=t L_{1}+(1-t) \tilde{L}=i \delta^{i} \partial_{i} \otimes 1_{m}+i 1_{p} \otimes[t \Phi(x)+(1-t) U(x)]
$$

$0 \leqq t \leqq 1$, is a homotopy of $L_{1}$ to $\tilde{L}$ within the class of Fredholm operators defined by the first part of the theorem. Thus $\tilde{L}$ has the same index as $L_{1}$ and $L$.

Remarks. Seeley's theorem tells us that the sufficient condition given in the corollary above for (1.3) to be Fredholm is also necessary. Thus, in particular, if it is not satisfied, the spectrum of $L^{\dagger} L$ and $L L^{\dagger}$ will be a continuous spectrum consisting of the entire positive real line.

To see how this corollary follows from Seeley's theorem, consider the symbol $\sigma(L)=\delta^{i} \xi_{i} \otimes 1_{m}+i 1_{p} \otimes \Phi(x)$. We have

$$
\sigma(L)^{\dagger} \sigma(L)=|\xi|^{2} \cdot 1+1_{p} \otimes|\Phi(x)|^{2}
$$

which shows that $|\sigma(L)|$ is bounded away from zero as $|x| \rightarrow \infty$ if and only if $|\Phi(x)|$ is. 
Finally note that the conclusions of the corollary do not change if we add an $L^{\infty}$ lower order term vanishing at $\infty$ to $L$.

\section{General First Order Operators}

We now derive some general formulas for first order elliptic operators with arbitrary coefficients. The crucial results are Proposition 1b) and Lemma 1. If certain conditions are satisfied, as described in the statements of the results, these formulas readily yield the index theorem. The proof of the latter in the next section consists, in fact, in verifying these conditions. We first give a general formula for the index of arbitrary operators on a Hilbert space.

Keeping in mind the setting described above, we consider an arbitrary operator $L$, which is assumed to be closed on a dense domain $D(L)$ in a Hilbert space $K$ which is the direct sum of $M$ copies of another Hilbert space $H$ : $K=\bigoplus_{i=1}^{M} H$. Then $L$ is a matrix of operators on $H: L=\left[L_{i j}\right], i, j=1, \ldots, M$. Each of the $L_{i j}$ is closed on a domain $D\left(L_{i j}\right) \subset H$. Now given an operator $A=\left[A_{i j}\right]$ on $K$ we define the internal trace of $A$, denoted by $\operatorname{tr} A$, to be the following operator on $H$ :

$$
\operatorname{tr} A=\sum_{i} A_{i i}
$$

with domain $\bigcap_{i=1}^{M} D\left(A_{i i}\right)$. The point of this definition is that although $A$ may not be trace-class on $K$, it is possible that $\operatorname{tr} A$ is trace-class on $H$. Thus we specify how a possibly undefined trace is to be calculated on $K$.

For $L$ as in the preceding paragraph, the operators $L L^{\dagger}$ and $L^{\dagger} L$ are selfadjoint and positive, where $L^{\dagger}$ is the Hilbert-space adjoint of $L$. If $z$ is not a nonnegative real number, $\left(L L^{\dagger}+z\right)^{-1}$ and $\left(L^{\dagger} L+z\right)^{-1}$ are bounded operators on $K$ and

$$
B_{z}=z \operatorname{tr}\left[\left(L^{\dagger} L+z\right)^{-1}-\left(L L^{\dagger}+z\right)^{-1}\right]
$$

is a bounded operator on $H$. Let

$$
f(z)=\operatorname{Tr} B_{z},
$$

where $\operatorname{Tr}$ denotes the trace in the Hilbert space $H$ : If $\left\{\phi_{k}\right\}_{k=1}^{\infty}$ is an orthonormal basis for $H, \operatorname{Tr} B=\sum_{k=1}^{\infty}\left(\phi_{k}, B \phi_{k}\right)$. Under certain assumptions, the index of $L$ is given in terms of the trace of $B_{z}$ on $H$, as described in the following lemma.

Lemma 1. Suppose $K, H, L, B_{z}$ are as above and furthermore $L: D(L) \rightarrow K$ is Fredholm and $B_{z}$ is trace-class on $H$, and $\operatorname{Tr}\left|B_{z}\right|$ is bounded for $z$ in a domain $C$ in the complex plane having $z=0$ as a limit point. Then

$$
\text { index }(L)=\lim _{z \rightarrow 0} f(z) \text {. }
$$

Proof. Since $L$ is Fredholm, $L^{\dagger} L$ and $L L^{\dagger}$ are Fredholm and the zero eigenvalues of $L^{\dagger} L$ and $L L^{\dagger}$ are isolated (see previous section). Obviously, $\operatorname{Ker}\left(L^{\dagger} L\right)=\operatorname{Ker}(L)$ and $\operatorname{Ker}\left(L L^{\dagger}\right)=\operatorname{Ker}\left(L^{\dagger}\right)$. Let $P_{+}$be the projection on $\operatorname{Ker}\left(L^{\dagger} L\right)$ and $P_{-}$the 
projection on $\operatorname{Ker}\left(L L^{\dagger}\right)$. Then the operator

$$
\tilde{B}_{z}=\operatorname{tr} \frac{z}{L^{\dagger} L+z}-\operatorname{tr} P_{+}-\operatorname{tr} \frac{z}{L L^{\dagger}+z}+\operatorname{tr} P_{-}=B_{z}-\operatorname{tr} P_{+}+\operatorname{tr} P_{-}
$$

is trace class since $B_{z}$ is and $P_{+}, P_{-}$are finite dimensional projections. Further, $\lim _{z \rightarrow 0} \tilde{B}_{z}=0$ strongly. Let $\left\{\phi_{k}\right\}_{k=1}^{\infty}$ be an orthonormal basis. Then the series

$$
\operatorname{Tr} \tilde{B}_{z}=\sum_{k=1}^{\infty}\left(\phi_{k}, \tilde{B}_{z} \phi_{k}\right)
$$

converges absolutely and uniformly for $z \in C$ and the limit of each term as $z \rightarrow 0$ is 0 .

Thus $\lim _{z \rightarrow 0} \operatorname{Tr} \tilde{B}_{z}=0$ and

$$
\lim _{z \rightarrow 0} f(z)=\lim _{z \rightarrow 0} \operatorname{Tr} \tilde{B}_{z}+\operatorname{Tr} P_{+}-\operatorname{Tr} P_{-}=\operatorname{index}(L) .
$$

The following lemma provides the bridge between the region $z \rightarrow 0$, where the index is computed according to Lemma 1 , and $z \rightarrow \infty$ where $\operatorname{Tr} B_{z}$ is computed explicitly as a function of $z$.

Lemma 2. With all the assumptions and definitions preceding Lemma 1, suppose $B_{z}$ is trace-class for $z$ in a domain $C$. Then $f(z)=\operatorname{Tr} B_{z}$ is analytic for $z \in C$.

Proof. Let $\left\{\phi_{k}\right\}_{k=1}^{\infty}$ be an orthonormal basis for $H$ and let

$$
f_{N}(z)=\sum_{k=1}^{N}\left(\phi_{k}, B_{z} \phi_{k}\right) \text {. }
$$

Then each $f_{N}(z)$ is analytic and $f_{N}(z)$ is bounded for all $N$ and all $z$ in a compact subset of $C$. Thus $f(z)=\lim _{N \rightarrow \infty} f_{N}(z)$ is analytic in $C$.

Proposition 1 below gives an identity (a) for arbitrary first order elliptic operators. This identity can be used under certain conditions to calculate the function $f(z)$ introduced above and, therefore, the index. That these conditions together with the assumptions of Lemmas 1 and 2 are satisfied by the operators of the kind introduced in (1.3) will be shown in the proof giving the index of these operators. The reader should not be distracted by the complicated formula (2.4) below, as it will in fact give zero contribution in the case that we study in the next section.

Proposition 1. Let $L$ be a first order elliptic differential operator on the Hilbert space $K=\bigoplus_{1}^{M} L^{2}\left(\mathbb{R}^{n}\right)$ :

$$
L=i \delta^{i}(x) \partial_{i}+i \Phi(x)
$$

where $\delta^{i}(x), \Phi(x)$ are $M \times M$ matrices of smooth functions. Let $B_{z}$ and $f(z)$ be defined by (2.1) and (2.2) respectively, and suppose $z$ is not a negative real number or 0 . Let $\delta^{i}$, $\Phi$ denote the multiplication operators by $\delta^{i}(x)$ and $\Phi(x)$. Then 
a) $B_{z}$ is an integral operator with kernel $B_{z}(x, y)$ which satisfies for $x \neq y$

$$
2 B_{z}(x, y)=\left(\frac{\partial}{\partial x^{i}}+\frac{\partial}{\partial y^{i}}\right) J_{z}^{i}(x, y)+A_{z}(x, y),
$$

where $J_{z}^{i}(x, y), A_{z}(x, y)$ are the kernels of the integral operators

$$
J_{z}^{i}=i \operatorname{tr}\left(L\left(L^{\dagger} L+z\right)^{-1} \delta^{i \dagger}\right)-i \operatorname{tr}\left(L^{\dagger}\left(L L^{\dagger}+z\right)^{-1} \delta^{i}\right)
$$

and

$$
\begin{aligned}
A_{z}= & i \operatorname{tr}\left[\delta^{i \dagger}, \partial_{i} L\left(L^{\dagger} L+z\right)^{-1}\right]-i \operatorname{tr}\left[\delta^{i}, \partial_{i} L^{\dagger}\left(L L^{\dagger}+z\right)^{-1}\right] \\
& -i \operatorname{tr}\left[\Phi, L^{\dagger}\left(L L^{\dagger}+z\right)^{-1}\right]-i \operatorname{tr}\left[\Phi^{\dagger}, L\left(L^{\dagger} L+z\right)^{-1}\right] \\
& +i \operatorname{tr}\left[\delta^{i}{ }_{, i}^{\dagger}, L\left(L^{\dagger} L+z\right)^{-1}\right],
\end{aligned}
$$

where $[A, B]=A B-B A, \delta^{i},{ }_{j}(x)=\frac{\partial \delta^{i}(x)}{\partial x^{j}}$.

b) Suppose that $B_{z}(x, y)$ is continuous near $x=y$ and the function $J_{z}^{i}(x, y)$ introduced in a) is finite and differentiable for all $x, y$ (even $x=y$ ). Then a) implies

$$
2 B_{z}(x, x)=\partial_{i} J_{z}^{i}(x, x)+A_{z}(x, x)
$$

and, if $B_{z}$ is trace-class,

$$
2 f(z)=\lim _{R \rightarrow \infty} \int_{S_{R}^{n-1}} J_{z}^{i}(x, x) d S_{i}+\int A_{z}(x, x) d^{n} x,
$$

where $S_{R}^{n-1}$ is the $(n-1)$-sphere of radius $R$ and $d S_{i}$ is the vectorial surface element on the sphere.

Proof. We work backwards the steps that led us to study the index problem for first order operators. Define

$$
H=\left(\begin{array}{cc}
0 & L \\
L^{\dagger} & 0
\end{array}\right)
$$

acting on $K \oplus K$ and the $2 M \times 2 M$ matrix

$$
\gamma=\left(\begin{array}{cc}
1_{M} & 0 \\
0 & -1_{M}
\end{array}\right)
$$

which anticommutes with every matrix of the form $\left(\begin{array}{lr}0 & A_{1} \\ A_{2} & 0\end{array}\right)$. Further let

$$
\alpha^{i}(x)=\left(\begin{array}{cc}
0 & \delta^{i}(x) \\
\delta^{i}(x)^{\dagger} & 0
\end{array}\right)
$$

Let $G_{\lambda}(x, y)$ represent the kernel of the integral operator representing the resolvent

$$
G_{\lambda}=(H+\lambda)^{-1} \text {. }
$$

Note that $H$ can be represented in the form

$$
H=i \alpha^{i}(x) \partial_{i}+i A(x),
$$

where

$$
A(x)=\left(\begin{array}{cc}
0 & \Phi(x) \\
-\Phi(x)^{\dagger}+\delta^{i},{ }_{i}(x)^{\dagger} & 0
\end{array}\right) .
$$


By elliptic regularity, $G_{\lambda}(x, y)$ is smooth in both $x$ and $y$ for $x \neq y$ since it satisfies the equations

$$
\begin{aligned}
& {\left[i \alpha^{i}(x) \frac{\partial}{\partial x^{i}}+i A(x)+\lambda\right] G_{\lambda}(x, y)=0,} \\
& \quad-i \frac{\partial}{\partial y^{i}}\left[G_{\lambda}(x, y) \alpha^{i}(y)\right]+G_{\lambda}(x, y)[i A(y)+\lambda]=0
\end{aligned}
$$

for $x \neq y$. We note that $G_{i \lambda}$ is given by

$$
G_{i \lambda}=\left(\begin{array}{cc}
-i \lambda & L \\
L^{\dagger} & -i \lambda
\end{array}\right)\left(\begin{array}{cc}
\left(L L^{\dagger}+\lambda^{2}\right)^{-1} & 0 \\
0 & \left(L^{\dagger} L+\lambda^{2}\right)^{-1}
\end{array}\right) .
$$

From this formula we easily see that

$$
B_{z}(x, y)=-i z^{1 / 2} \operatorname{tr}\left[\gamma G_{i z^{1 / 2}}(x, y)\right] .
$$

Adding $\left(2.5^{\prime}\right)$ to $(2.5)$ multiplying by $\gamma$ on the left, taking the trace, using the cyclic property $\operatorname{tr} A B=\operatorname{tr} B A$ and noting that $\gamma$ anticommutes with $\alpha^{i}(x)$ and $A(x)$ we get

$$
2 B_{z}(x, y)=\left(\frac{\partial}{\partial x^{i}}+\frac{\partial}{\partial y^{i}}\right) J_{z}^{i}(x, y)+A_{z}(x, y),
$$

where

$$
J_{z}^{i}(x, y)=-i \operatorname{tr}\left(\gamma \alpha^{i}(y) G_{i z^{1 / 2}}(x, y)\right)
$$

and

$$
\begin{aligned}
A_{z}(x, y)= & -i \operatorname{tr}\left\{\gamma\left(\alpha^{i}(x)-\alpha^{i}(y)\right) \frac{\partial}{\partial x^{i}} G_{i z^{1 / 2}}(x, y)\right\} \\
& -i \operatorname{tr}\left\{\gamma(A(x)-A(y)) G_{i z^{1 / 2}}(x, y)\right\} .
\end{aligned}
$$

Using (2.6) and computing the trace over the block form we find the formulas (2.3) and (2.4).

Remark. The conditions of Proposition 1b) can be relaxed as follows. Instead of assuming that $J_{z}^{i}(x, y)$ is differentiable we may rather just require that $J_{z}^{i}(x, y)$ $+\sum_{\lambda} \alpha_{\lambda} J_{z \lambda}^{i}(x, y)$ is differentiable $\left(C^{1}\right)$ for some $z_{\lambda}, \alpha_{\lambda}$ (depending on $z, z_{\lambda}$ ) [11]. Then assuming the remaining conditions are satisfied the equations in Proposition 1b) are replaced by linear combinations of such equations with the coefficients above.

Finally we note that essentially the same equations can be written on an arbitrary orientable manifold by letting $B_{z}(x, x) \rightarrow B_{z}(x, x) d^{n} x, \quad A_{z}(x, x)$ $\rightarrow A_{z}(x, x) d^{n} x, \partial_{i} J_{z}^{i}(x, x) \rightarrow d J_{z}(x, x)$ for a suitable $(n-1)$-form $J_{z}(x, x)$.

\section{An Index Formula for Dirac Operators}

The general Proposition of the preceding Section is of particular practical value in the case of Dirac operators. As the simplest application we derive an index 
formula for the operator in (1.3) by showing that the conditions in Proposition 1b) are satisfied and then using the identity for the function $f(z)$ given there; in fact we find the explicit form of this function. The result is stated in Theorem 2 below, whose proof rests on Lemmas 4 and 5. The note following this article discusses how our result fits in the context of a more general index theory on $R^{n}$.

Theorem 2. Let $L$ be a first order differential operator on $\mathbb{R}^{n}, n$ odd, which up to $L^{\infty}$ zero order terms vanishing at $\infty$ is of the form

$$
L=i \delta^{i} \partial_{i} \otimes 1_{m}+i 1_{p} \otimes \Phi(x),
$$

where the $\delta^{i}$ are constant $p \times p$ matrices, $p=2^{(n-1) / 2}$, satisfying the algebra

$$
\delta^{i} \delta^{j}+\delta^{j} \delta^{i}=2 \delta^{i j} \cdot 1_{p}
$$

(thus $p$ is minimal for this algebra to be satisfied), $\Phi(x)$ is an $m \times m$ Hermitian matrix of $C^{\infty}$-functions such that $|\Phi(x)| \geqq B>0$ for $|x| \geqq C, B, C$ constants; further $\Phi(x)$ is asymptotically homogeneous of order 0 as $x \rightarrow \infty$. Let $U(x)=|\Phi(x)|^{-1} \Phi(x)$. Then the index of $L$ is given by

$$
\text { index }(L)=\frac{1}{2\left(\frac{n-1}{2}\right) !}\left(\frac{\mathrm{i}}{8 \pi}\right)^{\frac{n-1}{2}} \lim _{R \rightarrow \infty} \int_{S_{R}^{n-1}} \operatorname{tr}\left[U(x)(d U(x))^{n-1}\right] \text {, }
$$

where $(d U)^{n-1}$ is the $(n-1)$ st power of the matrix $d U$ with the differentials being multiplied by exterior multiplication; $S_{R}^{n-1}$ is the $(n-1)$-sphere of radius $R$.

Remarks. In the proof below we will obtain index $(L)$ as $f(0)$ where $f(z)$ is defined by (2.2). If $\Phi$ in the definition of $L$ above is unitary, we will prove the following simple explicit formula for $f(z)$ :

$$
f(z)=\frac{1}{(1+z)^{n / 2}} \text { index }(L) .
$$

The formula (3.2) for the index remains essentially the same if $n$ is even, and it gives trivially that index $(L)=0$ in that case, for any $L$ of the form (3.1).

Proof of the Theorem. We prove the theorem using Proposition 1b). The crucial Lemma 4 below depends heavily on the algebra satisfied by the $\delta^{i}$. That algebra alone implies the following important facts:

A) If $n$ and $k$ are odd and $k<n$,

$$
\operatorname{tr}\left(\delta^{i_{1}} \ldots \delta^{i_{k}}\right)=0 \text {. }
$$

If $n$ is even, (3.4) is true for all odd $k$.

B) If $n$ is odd and the order of the matrices $\delta^{i}$ is $2^{(n-1) / 2}$, i.e. the minimal one required to satisfy the algebra $\delta^{i} \delta^{j}+\delta^{j} \delta^{i}=2 \delta^{i j}$, we have

$$
\operatorname{tr}\left(\delta^{i_{1}} \ldots \delta^{i_{n}}\right)=(2 i)^{\frac{n-1}{2}} \varepsilon^{i_{1} \ldots i_{n}},
$$

where $\varepsilon^{i_{1} \ldots i_{n}}$ is the fully antisymmetric symbol in $n$ diemsions. (3.5) is proved by using a representation of the $\delta^{\imath}$ in dimension $n$ in terms of those in dimension $n-2$. 
These properties of the matrices $\delta^{i}$ will be responsible for the cancellation of the singularities in the traces that we use in the proof of the theorem.

In Proposition 1 we invariably encounter the objects $\left(L L^{\dagger}+z\right)^{-1}$ and $\left(L^{\dagger} L+z\right)^{-1}$. We first obtain a useful form for them. We have

$$
\begin{aligned}
& L L^{\dagger}=-\partial^{2} \cdot 1+\delta^{i} \otimes \Phi_{, i}+1_{p} \otimes \Phi^{2} \\
& L^{\dagger} L=-\partial^{2} \cdot 1-\delta^{i} \otimes \Phi_{, i}+1_{p} \otimes \Phi^{2} .
\end{aligned}
$$

To proceed we need the following

Lemma 3. Suppose that $V(x)=A^{i},{ }_{i}(x)$ where $A_{i}(x)$ are Hermitian matrices of bounded functions and the derivative is taken in the sense of distributions. Let $V$ be the multiplication operator by $V(x)$. Let $Q_{z}=\left(-\partial^{2}+z\right)^{-1}$ where $\operatorname{Re} z>0$. Then there is a unique bounded operator $W_{z}$ such that $\left(\phi, W_{z} \psi\right)=\left(Q_{z}^{1 / 2} \phi, V Q_{z}^{1 / 2} \psi\right)$, whenever $Q_{\bar{z}}^{1 / 2} \phi, Q_{z}^{1 / 2} \psi \in \mathscr{S}\left(\mathbb{R}^{n}\right)$. (Here $Q_{z}^{1 / 2}$ is defined by the spectral theorem.) In other words $Q_{\bar{z}}^{1 / 2} V Q_{z}^{1 / 2}$ extends uniquely to a bounded operator on the space of $L^{2}$-vectors. Furthermore $W_{z}^{\dagger}=W_{\bar{z}}$ and $\left\|W_{z}\right\|<\frac{1}{(\operatorname{Re} z)}\|A\|$ where $\|A\|=\sup _{x, i}\left\|A^{i}(x)\right\|$ and $\left\|A^{i}(x)\right\|$ is the norm of $A^{i}(x)$ as a finite dimensional linear transformation.

Proof. Let $\phi, \psi \in \mathscr{S}\left(\mathbb{R}^{n}\right)$. Then by the definition of $V$,

$$
(\phi, V \psi)=-\int\left[\partial_{i} \phi(x)^{\dagger} A^{i}(x) \psi(x)+\phi(x)^{\dagger} A^{i}(x) \partial_{i} \psi(x)\right]
$$

which implies the estimate

$$
\begin{aligned}
|(\phi, V \psi)| & \leqq\|A\| \cdot \int \sum_{i}\left(\left\|\partial_{i} \phi(x)\right\| \cdot\|\psi(x)\|+\|\phi(x)\| \cdot\left\|\partial_{i} \psi(x)\right\|\right) \\
& \leqq \frac{1}{\operatorname{Re} z}\|A\| \cdot\left(\phi,\left(-\partial^{2}+\operatorname{Re} z\right) \phi\right)^{1 / 2}\left(\psi,\left(-\partial^{2}+\operatorname{Re} z\right) \psi\right)^{1 / 2} .
\end{aligned}
$$

Now $-\partial^{2}+\operatorname{Re} z \leqq\left(-\partial^{2}+\bar{z}\right)^{1 / 2}\left(-\partial^{2}+z\right)^{1 / 2}$, so that we obtain the estimate

$$
|(\phi, V \psi)| \leqq \frac{1}{\operatorname{Re} z}\|A\|\left(\phi, Q_{z}^{-1 / 2} Q_{\bar{z}}^{-1 / 2} \phi\right)^{1 / 2} \cdot\left(\psi, Q_{\bar{z}}^{-1 / 2} Q_{z}^{-1 / 2} \psi\right)^{1 / 2} .
$$

Letting $\phi=Q_{\bar{z}}^{1 / 2} \phi^{\prime}, \psi=Q_{z}^{1 / 2} \psi^{\prime}$ we see that $\left(Q_{\bar{z}}^{1 / 2} \cdot V Q_{z}^{1 / 2} \cdot\right)$ is a bounded sesquilinear form on a dense subspace of the Hilbert space, so that $W_{z}$ is defined uniquely. The estimate on the norm of $W_{z}$ follows from the estimate on $(\phi, V \psi)$ above.

Returning now to the proof of the theorem let $\operatorname{Re} z>0$, and

$R_{z}=\left(-\partial^{2} \cdot 1+1_{p} \otimes \Phi^{2}+z \cdot 1\right)^{-1}$.

$R_{z}$ is compared with $Q_{z}=\left(-\partial^{2}+z\right)^{-1}$. Both $R_{z}$ and $Q_{z}$ are integral operators with kernels $R_{z}(x, y), Q_{z}(x, y)$. We have the following simple facts

(a) $\left\|R_{z}\right\| \leqq\left\|Q_{z}\right\| \leqq \frac{c}{\operatorname{Re} z} \quad\left\|R_{z} Q_{z}^{-1}\right\| \leqq c^{\prime}$,

(b) $Q_{z}(x, y)=\frac{1}{(2 \pi)^{n}} \int d^{n} p \frac{e^{i(x-y) p}}{p^{2}+z}$. 
Thus if $z$ is real and positive, $Q_{z}(x, y)=Q_{z}(x-y)=(\sqrt{z})^{n-2} Q_{1}(\sqrt{z}(x-y))$

(c) $0<\left|R_{z}(x, y)\right| \leqq\left|Q_{z}(x, y)\right| \leqq K \cdot \frac{e^{-\operatorname{Re} \sqrt{z}|x-y|}}{|x-y|^{n-2}}$.

$Q_{z}(x, y)$ is $C^{\infty}$ away from $x=y$. Near $x=y$ it behaves like $|x-y|^{-n+2} \cdot R_{z}(x, y)$ behaves similarly. The singularities of $R_{z}(x, y)$ are conveniently studied by the expansion

$$
R_{z}=Q_{z}+\left(-Q_{z} \Phi^{2}\right) Q_{z}+\ldots+\left(-Q_{z} \Phi^{2}\right)^{k} R_{z}
$$

in which the $r^{\text {th }}$ term blows up like $|x-y|^{-n+1+r}$ near $x=y$.

Now in terms of $R_{z}$ define the operator

$$
Z_{z}=R_{z}^{1 / 2}\left[\delta^{i} \otimes \Phi_{, i}\right] R_{z}^{1 / 2}
$$

which is bounded by Lemma 3, with $\left\|Z_{z}\right\|<\frac{C}{\operatorname{Re} z}$. For $\operatorname{Re} z$ large enough we then have

$$
\begin{aligned}
& \left(L L^{\dagger}+z\right)^{-1}=R_{z}^{1 / 2}\left(1+Z_{z}\right)^{-1} R_{z}^{1 / 2}=R_{z}^{1 / 2} \sum_{k=0}^{\infty}(-1)^{k} Z_{z}^{k} R_{z}^{1 / 2} \\
& \left(L^{\dagger} L+z\right)^{-1}=R_{z}^{1 / 2}\left(1-Z_{z}\right)^{-1} R_{z}^{1 / 2}=R_{z}^{1 / 2} \sum_{k=0}^{\infty} Z_{z}^{k} R_{z}^{1 / 2} .
\end{aligned}
$$

We are now ready to prove

Lemma 4. Let $L$ be as in (3.1) and $J_{z}^{i}(x, y) A_{z}(x, y)$ be defined as in Proposition 1. For $\operatorname{Re} z$ positive and large enough:

a) If $n=1, J_{z}^{i}(x, y)-J_{z^{\prime}}^{i}(x, y)$ is differentiable near $y=x$ for any $z^{\prime}$ with $\operatorname{Re} z^{\prime}>0$.

b) If $n=3, J_{z}^{i}(x, y)+\alpha J_{z^{\prime}}^{i}(x, y)+\beta J_{z^{\prime \prime}}^{i}(x, y)$ is differentiable near $y=x$, where

$$
z^{\prime} \neq z^{\prime \prime}, \operatorname{Re} z^{\prime}, \operatorname{Re} z^{\prime \prime}>0 \text { and } \alpha=\frac{z^{\prime \prime}-z}{z^{\prime}-z^{\prime \prime}} \beta=\frac{z-z^{\prime}}{z^{\prime}-z^{\prime \prime}} \text {. }
$$

c) If $n \geqq 5, J_{z}^{i}(x, y)$ is differentiable.

For all $n, \lim _{y \rightarrow x} A_{z}(x, y)=0$, and $J_{z}^{i}(x, y)$ is continuous in $x$ and $y$.

Proof. From (2.3) we see that $J_{z}^{i}(x, y)$ is the kernel of the integral operator.

$$
J_{z}^{i}=i \operatorname{tr}\left[\delta^{i} L^{\dagger}\left(L L^{\dagger}+z\right)^{-1}\right]-i \operatorname{tr}\left[\delta^{i} L\left(L^{\dagger} L+z\right)^{-1}\right] .
$$

Using (3.7) we obtain

$$
\begin{aligned}
J_{z}^{i}= & -i \operatorname{tr}\left[\delta^{i}\left(L+L^{\dagger}\right) R_{z}^{1 / 2} \sum_{k=0}^{\infty} Z_{z}^{2 k+1} R_{z}^{1 / 2}\right] \\
& -i \operatorname{tr}\left[\delta^{i}\left(L-L^{\dagger}\right) R_{z}^{1 / 2} \sum_{k=0}^{\infty} Z_{z}^{2 k} R_{z}^{1 / 2}\right] .
\end{aligned}
$$


Now $L+L^{\dagger}=2 i \delta^{i} \partial_{i} \otimes 1_{m}, L-L^{\dagger}=2 i 1_{p} \otimes \Phi(x)$. Using the trace identities (3.4) we see that both series above start with a high power of $Z_{z}$. If $n \geqq 3$,

$$
\begin{aligned}
J_{z}^{i}= & 2 \operatorname{tr}\left[\left(\delta^{i} \delta^{j} \partial_{j} \otimes 1_{m}\right) R_{z}^{1 / 2} \sum_{k=0}^{\infty} Z_{z}^{n+2 k-2} R_{z}^{1 / 2}\right] \\
& +2 \operatorname{tr}\left[\left(\delta^{i} \otimes \Phi\right) R_{z}^{1 / 2} \sum_{k=0}^{\infty} Z_{z}^{n+2 k-1} R_{z}^{1 / 2}\right]
\end{aligned}
$$

If $n=1$, the full series (3.8) contributes to $J_{z}^{i}$. Using the expansion of $R_{z}$ in terms of $Q_{z}$ referred to above, we can study the regularity of each term in (3.9) near $y=x$. For $n \geqq 5$ we find that all terms are $C^{1}$ but for $n=1$ or $n=3$ the lowest terms in (3.9) exhibit singularities which can be cancelled by forming linear combinations of $J_{z}$ 's with different $z$ 's. It is straightforward to check a) and b).

The statement about $A_{z}(x, y)$ follows if we note that in our case

$$
\begin{aligned}
A_{z}= & i \operatorname{tr}\left[\left(1_{p} \otimes \Phi\right), L^{\dagger}\left(L L^{\dagger}+z\right)^{-1}\right] \\
& +i \operatorname{tr}\left[\left(1_{p} \otimes \Phi\right), L\left(L^{\dagger} L+z\right)^{-1}\right]
\end{aligned}
$$

and use (3.7) again. The singularities of the resolvents disappear and $\lim _{y \rightarrow x} A_{z}(x, y)=0$ follows from the presence of $\Phi(y)-\Phi(x)$.

Lemma 5. Let $B_{z}$ be defined by (2.1) where $L$ is given by (3.1). Then for $\operatorname{Re} z>0, B_{z}$ is trace-class and $\operatorname{Tr}\left|B_{z}\right|$ is bounded uniformly on compact subsets of the domain $\operatorname{Re} z>0$. Furthermore, $\lim _{\operatorname{Re} z \rightarrow \infty} \operatorname{Tr}\left|B_{z}\right|=0$.

Proof. We use (3.7) to find

$$
\begin{aligned}
B_{z} & =2 z \operatorname{tr}\left[R_{z}^{1 / 2} \frac{Z_{z}}{1-Z_{z}^{2}} R_{z}^{1 / 2}\right] \\
& =2 z \sum_{k=0}^{\frac{n-1}{2}} \operatorname{tr}\left[R_{z}^{1 / 2} Z_{z}^{2 k+1} R_{z}^{1 / 2}\right]+2 z \operatorname{tr}\left[R_{z}^{1 / 2} \frac{Z_{z}^{n+2}}{1-Z_{z}^{2}} R_{z}^{1 / 2}\right] .
\end{aligned}
$$

Each term, except for the last one, in the sum is identically zero because of (3.4). It only remains therefore to estimate the trace of

$$
\operatorname{tr}\left[R_{z}^{1 / 2} Z_{z}^{n} R_{z}^{1 / 2}\right]+\operatorname{tr}\left[R_{z}^{1 / 2} \frac{Z_{z}^{n+2}}{1-Z_{z}^{2}} R_{z}^{1 / 2}\right] .
$$

We estimate each of these terms separately.

(a) $\operatorname{tr}\left[R_{z}^{1 / 2} Z_{z}^{n} R_{z}^{1 / 2}\right]=\int d x d x_{1} \ldots d x_{n} R_{z}\left(x, x_{1}\right) R_{z}\left(x_{1}, x_{2}\right)$

$$
\ldots R_{z}\left(x_{n}, x\right) \varepsilon^{i_{1} \ldots i_{n}} \operatorname{tr}\left[\partial_{i_{1}} \Phi\left(x_{1}\right) \ldots \partial_{i_{n}} \Phi\left(x_{n}\right)\right] \text {. }
$$

To merely indicate how the estimate that is quoted below for this quantity comes about, assume that $\Phi(x)$ depends everywhere only on $\frac{x}{|x|}$. Now the integral can be estimated by one of the same form with $R_{z}$ replaced by $Q_{z}$ and $z$ by $(\operatorname{Re} \sqrt{z})^{2}$. The 
latter is then equal to $(\operatorname{Re} \sqrt{z})^{-n-2} I$, where $I$ is a finite constant. To see this, rescale the variables of integration according to $x \rightarrow \frac{x}{\operatorname{Re} \sqrt{z}}$, and to verify the convergence of the integral change variables by $x_{i} \rightarrow x+y_{i}$. The $y_{i}$-integrals converge because of the exponential behavior of $Q_{z}$. The $x$-integral converges because as $x \rightarrow \infty$,

$$
\varepsilon^{i_{1} \ldots i_{n}} \operatorname{tr}\left[\partial_{i_{1}} \Phi\left(x+y_{1}\right) \ldots \partial_{i_{n}} \Phi\left(x+y_{n}\right)\right]
$$

goes to zero faster than $|x|^{-n}$. In fact, it tends to zero at least as fast as $|x|^{-n}$ because $\partial_{i} \Phi(x) \sim \frac{1}{|x|}$, but note that as $|x| \rightarrow \infty$ the arguments of the $\Phi$ 's become approximately equal while the presence of the $\varepsilon$-symbol guarantees that

$$
\varepsilon^{i_{1} \ldots i_{n}} \operatorname{tr}\left[\partial_{i_{1}} \Phi(x) \ldots \partial_{i_{n}} \Phi(x)\right]=0
$$

identically, so that (3.12) actually approaches zero faster than $|x|^{-n}$.

Thus we conclude

$$
\operatorname{Tr}\left|\operatorname{tr} R_{z}^{1 / 2} Z_{z}^{n} R_{z}^{1 / 2}\right| \leqq(\operatorname{Re} \sqrt{z})^{-n-2} I .
$$

(b) For the second term in (3.11) we note that

$$
\begin{aligned}
\operatorname{Tr}\left|R_{z}^{1 / 2} \frac{Z_{z}^{n+2}}{1-Z_{z}^{2}} R_{z}^{1 / 2}\right| & \leqq \operatorname{Tr}\left|Z_{z}^{n+2}\right| \cdot\left\|R_{z}^{1 / 2} \frac{1}{1-Z_{z}^{2}} R_{z}^{1 / 2}\right\| \\
& =\left\|\frac{1}{L L^{\dagger}+z}+\frac{1}{L^{\dagger} L+z}\right\| \cdot \operatorname{Tr}\left|Z_{z}^{n+2}\right| \leqq \frac{2}{\operatorname{Re} z} \operatorname{Tr}\left|Z_{z}^{n+2}\right|
\end{aligned}
$$

Now $\operatorname{Tr}\left|Z_{z}^{n+2}\right| \leqq \operatorname{Tr}\left[Q_{\operatorname{Re} z}^{1 / 2}\left|\delta^{i} \otimes \Phi_{, i}\right| Q_{\mathrm{Re} z}^{1 / 2}\right]^{n+2}$ as can be seen from the integral representation of the trace. Now use the estimate, for positive operators $A, B$ [12]

$$
\operatorname{Tr}\left(B^{1 / 2} A B^{1 / 2}\right)^{m} \leqq \operatorname{Tr}\left(B^{m / 2} A^{m} B^{m / 2}\right)
$$

to get

$$
\operatorname{Tr}\left|Z_{z}^{n+2}\right| \leqq \operatorname{Tr}\left(Q_{\operatorname{Re} z}^{n+2}\left|\delta^{i} \otimes \Phi_{, i}\right|^{n+2}\right)
$$

which is finite (writing the trace in Fourier space). In fact, we get

$$
\operatorname{Tr}\left|Z_{z}^{n+2}\right| \leqq(\operatorname{Re} z)^{-n-2} I^{\prime}
$$

so that

$$
\operatorname{Tr}\left|R_{z}^{1 / 2} \frac{Z_{z}^{n+2}}{1-Z_{z}^{2}} R_{z}^{1 / 2}\right| \leqq \frac{2}{(\operatorname{Re} z)^{n+3}} I^{\prime} .
$$

The results of (a) and (b) establish the lemma.

We are now ready to apply Proposition 1 and Lemma 1 to prove the theorem. For convenience we make the replacement $\Phi(x) \rightarrow U(x)=|\Phi(x)|^{-1} \Phi(x)$ outside a compact region containing the zeros of $\Phi(x)$. It is then understood that we take the limit as this region becomes a set of discrete points. This is legitimate because of the uniform convergence of the integrals involved in the final answer. Note that in this limit $R_{z} \rightarrow Q_{z+1}$. Lemma 4 tells us that Proposition 1 applies and

$$
2 f(z)=\lim _{R \rightarrow \infty} \int_{S_{R}^{n-1}} d S^{i} J_{z}^{i}(x, x)-2 \sum_{\lambda} \alpha_{\lambda} f\left(z_{\lambda}\right)+\sum_{\lambda} \lim _{R \rightarrow \infty} \int_{S_{R}^{n-1}} d S_{i} \alpha_{\lambda} J_{x_{\lambda}}^{i}(x, x),
$$


where the constants $\alpha_{\lambda}$ are specified for the various dimensions by Lemma 4 . So we need to compute $\lim _{R \rightarrow \infty} d S_{i} J_{z}^{i}(x, x)$ as an $(n-1)$-form on $S_{1}^{n-1}$. Thus only terms that behave like $|x|^{-n+1}$ are significant in $J_{z}^{i}(x, x)$. We determine them using (3.9). It is easy to check that the $k=0$ terms in the infinite series in that equation are the only ones that have an $|x|^{-n+1}$ behavior, while the remaining ones vanish at infinity faster than $|x|^{-n+1}$. Thus two terms are relevant.

(1) $2 \operatorname{tr}\left[\left(\delta^{i} \delta^{j} \partial_{j} \otimes 1_{m}\right) Q_{z+1}^{1 / 2} Z_{z}^{n-2} Q_{z+1}^{1 / 2}\right](x, x)$

$$
\begin{aligned}
= & \lim _{y \rightarrow x} 2 \operatorname{tr}\left[\left(\delta^{i} \delta^{j} \frac{\partial}{\partial x^{j}} \otimes 1_{m}\right) \int d x_{1} \ldots d x_{n-2} Q_{z+1}\left(x, x_{1}\right)\right. \\
& \left.\cdot\left(\delta^{i_{1}} \otimes U_{,_{1}}\left(x_{1}\right)\right) \ldots\left(\delta^{i_{n-2}} \otimes U_{i_{n-2}}\left(x_{n-2}\right)\right) Q_{z+1}\left(x_{n-2}, y\right)\right] .
\end{aligned}
$$

A simple integration by parts on $x_{1}$ together with the fact that $\operatorname{tr} \delta^{i} \delta^{j} \delta^{i_{1}}, \ldots, \delta^{i_{n-2}}$ $=(2 i)^{(n-1) / 2} \varepsilon^{i j i_{1} \ldots i_{n-2}}$ shows that the contribution from this term is zero.

(2) $2 \operatorname{tr}\left[\left(\delta^{i} \otimes U(x)\right) Q_{z+1}^{1 / 2} Z_{z}^{n-1} Q_{z+1}^{1 / 2}\right](x, x)$

$$
\begin{aligned}
= & 2 \operatorname{tr}\left[\left(\delta^{i} \otimes U(x)\right) \int d x_{1} \ldots d x_{n-1} Q_{z+1}\left(x, x_{1}\right)\right. \\
& \cdot\left(\delta^{i_{1}} \otimes U_{i_{1}}\left(x_{1}\right)\right) \ldots \delta^{i_{n-1}} \otimes U_{i_{n-1}}\left(x_{n-1}\right) \cdot Q_{z+1}\left(x_{n-1}, x\right) .
\end{aligned}
$$

Changing variables of integration to $y_{i}=x-y_{i}$, we see that to lowest order in $|x|^{-1}$ this is

$$
\begin{aligned}
& 2(2 i)^{\frac{n-1}{2}} \varepsilon^{i i_{1} \ldots i_{n-1}} \operatorname{tr}\left[U(x) \partial_{i_{1}} U(x) \ldots \partial_{i_{n-1}} U(x)\right] \\
& \quad \cdot \int d y_{1} \ldots d y_{n-1} Q_{z+1}\left(\left|y_{1}\right|\right) Q_{z+1}\left(\left|y_{2}-y_{1}\right|\right) \ldots Q_{z+1}\left(\left|y_{n-1}\right|\right) .
\end{aligned}
$$

The integral can be evaluated easily using Fourier transform; it is equal to

$$
\frac{1}{(2 \pi)^{n}} \int(d p) \frac{1}{\left(p^{2}+z+1\right)^{n}}=\frac{1}{2^{2(n-1)+1}} \frac{1}{\left(\frac{n-1}{2}\right) !} \frac{1}{\pi^{(n-1) / 2}} \cdot \frac{1}{(1+z)^{n / 2}} .
$$

Thus we see that the quantity on the right-hand side of (3.2) has appeared as a $z$ independent factor in $\int d S_{i} J_{z}^{i}(x, x)$. Call that quantity $N$. We have obtained for the $J_{z}^{i}$ constructed from $L$ of (3.1) with the replacement $\Phi(x) \rightarrow U(x)$

$$
\int d S_{i} J_{z}^{i}(x, x)=\frac{1}{(1+z)^{n / 2}} 2 N
$$

so that, by (3.13),

$$
2 f(z)=\frac{1}{(1+z)^{n / 2}} 2 N+\sum_{\lambda} \alpha_{\lambda} \frac{1}{\left(1+z_{\lambda}\right)^{n / 2}} 2 N-2 \sum_{\lambda} \alpha_{\lambda} f\left(z_{\lambda}\right) .
$$

Now take the limit $\operatorname{Re} z_{\lambda} \rightarrow \infty$ and use Lemma 4 for the $\alpha_{\lambda}$ and Lemma 5 for $\lim _{\operatorname{Re} z_{\lambda} \rightarrow \infty} f\left(z_{\lambda}\right)$ to get

$$
f(z)=\frac{1}{(1+z)^{n / 2}} N
$$


C. Callias

which proves (3.3) for $\Phi(x)$ unitary and $\operatorname{Re} z$ large. Now use Lemma 2 to continue to $z \rightarrow 0$ and get (3.2) by Lemma 1 .

Example: $n=1$. To illustrate our results and how our approach actually goes beyond merely providing a formula for the index, we consider the case $n=1$. The operator (3.1) is now of the form

$$
L=i \frac{d}{d x}+i A(x)
$$

where $A(x)$ is $C^{\infty}$ hermitian matrix valued function on $R$. Besides the index formula, a relation of the type (3.3) is valid for arbitrary $A(x)$ bounded away from 0 as $|x| \rightarrow \infty$ :

$$
\operatorname{Tr}\left(\frac{z}{L^{\dagger} L+z}-\frac{z}{L L^{\dagger}+z}\right)=-\frac{1}{2}\left[\frac{A(+\infty)}{\left[z+A(+\infty)^{2}\right]^{1 / 2}}-\frac{A(-\infty)}{\left[z+A(-\infty)^{2}\right]^{1 / 2}}\right]
$$

if we assume that the limits $\lim _{x \rightarrow+\infty} A(x)=A(+\infty) \lim _{x \rightarrow-\infty} A(x)=A(-\infty)$ exist and are finite. Thus in particular [13]

$$
\operatorname{index}(L)=-\frac{1}{2}\left[\frac{A(+\infty)}{|A(+\infty)|}-\frac{A(-\infty)}{|A(-\infty)|}\right]
$$

We see that, a bit surprisingly, the quantity on the left-hand side of (3.14) shares with the index the property of invariance under $L$-compact perturbations.

The simplest case we can consider is with only one internal degree of freedom. The index can be determined by explicit solution. Let

$$
L=i \frac{d}{d x}+i \phi(x)
$$

$\lim _{x \rightarrow+\infty} \phi(x)=\phi_{+}, \lim _{x \rightarrow-\infty} \phi(x)=\phi_{-}$. Solving

$$
\begin{aligned}
& L \psi_{-}=0 \\
& L \psi_{+}=0
\end{aligned}
$$

we get

$$
\begin{aligned}
& \psi_{-}(x)=A_{-} e^{-\int_{0}^{x} \phi\left(x^{\prime}\right) d x^{\prime}} \\
& \psi_{+}(x)=A_{+} e^{+\int_{0}^{x} \phi\left(x^{\prime}\right) d x^{\prime}} .
\end{aligned}
$$

Then $\int\left|\psi_{-}\right|^{2}<\infty$ if and only if $\phi_{+}>0, \phi_{-}<0$ and $\int\left|\psi_{+}\right|^{2}<\infty$ if $\phi_{+}<0, \phi_{-}>0$, while neither is integrable otherwise. This checks with formula (3.15) for the index.

In the more explicit instance

$$
\phi(x)=\alpha \varepsilon(x)= \begin{cases}\alpha, & x>0 \\ -\alpha & x<0\end{cases}
$$


we can compute

$$
\operatorname{Tr}\left(\frac{z}{L^{\dagger} L+z}-\frac{z}{L L^{\dagger}+z}\right)=\int_{-\infty}^{\infty} d x\left[G_{z}^{\alpha}(x, x)-G_{z}^{-\alpha}(x, x)\right],
$$

where $G_{z}^{\alpha}(x, y)$ solves

$$
\left[-\frac{d^{2}}{d x^{2}}-\alpha \delta(x)+\alpha^{2}+z\right] G_{z}^{\alpha}(x, y)=\delta(x-y) .
$$

Let $z^{\prime}=\left(\alpha^{2}+z\right)^{1 / 2}$. We find

$$
G_{z}^{\alpha}(x, y)=\frac{1}{2\left(z^{\prime}-\alpha\right)} e^{-\sqrt{z^{\prime}}|x-y|}+\frac{\alpha}{2 z^{\prime}\left(z^{\prime}-\alpha\right)} \theta(x y)\left(e^{-\sqrt{z^{\prime}}|x|} e^{-\sqrt{z^{\prime}}|y|}-e^{-\sqrt{z^{\prime}}|x-y|}\right),
$$

where $\theta(x)=1$ for $x>0$ and 0 for $x<0$. This gives

$$
\operatorname{Tr}\left(\frac{z}{L^{\dagger} L+z}-\frac{z}{L L^{\dagger}+z}\right)=\frac{\alpha}{\left(\alpha^{2}+z\right)^{1 / 2}} .
$$

This checks with (3.14). [Although (3.16) is not $C^{\infty}$, our results still apply by a limiting argument.]

\section{Application: Fermions in the Field of SU(2) Monopoles}

As an application of the open space theorem that we derived, we study the Dirac equation for an isospin- $T$ particle in the field of a static (time-independent) system of SU(2)-magnetic monopoles. More precisely, we have here $n=3$, the dimension of space in ordinary four-dimensional Minkowski space-time and the potentials in (1.1) are given by

$$
\begin{aligned}
& A_{i}(x)=g T^{\alpha} A_{a}^{i}(x) \\
& \Phi(x)=\mu \phi^{a}(x) T^{a}+m,
\end{aligned}
$$

where $g, \mu, m$ (the mass of the fermion) are constants and the index $a$ runs from 1 to 3. The $T^{a}$ are the generators of isospin rotations. They satisfy

$$
\begin{aligned}
{\left[T^{a}, T^{b}\right] } & =i \varepsilon^{a b c} T^{c} \\
T^{a} T^{a} & =T(T+1) \cdot 1 .
\end{aligned}
$$

$A_{a}^{\mu}, \phi^{a}$ are the gauge potentials and Higgs fields of an $\mathrm{SO}(3)$ Yang-Mills theory coupled with Higgs fields [ $\mu$ is a space-time index and $a$ is the $\mathrm{SO}(3)$ index]. The static configuration

$$
\begin{aligned}
A_{a}^{0} & =0 \\
A_{a}^{i} & =A_{a}^{i}(x) \\
\phi^{a} & =\phi^{a}(x)
\end{aligned}
$$

could arise as a static finite energy solution of the coupled Yang-Mills-Higgs equations in the absence of fermions [14]. $A_{a}^{i}(x)$ are bounded and vanish at $\infty$, 
while $\phi^{a}(x) \phi^{a}(x) \rightarrow 1$ as $x \rightarrow \infty$. In a suitable interpretation, this configuration represents a system of magnetic charges with total magnetic charge [15]

$$
N=-\frac{1}{8 \pi} \int_{S_{\infty}^{2}} \varepsilon_{a b c} \phi^{a} d \phi^{b} d \phi^{c}
$$

essentially the degree of $\phi^{a}$ regarded as a mapping $S_{\infty}^{2} \rightarrow S_{1}^{2}$. Actually the only such configuration that is known as a solution of the Yang-Mills equation is the spherically symmetric one with unit magnetic charge [14]. It is not known whether more complicated static solutions exist.

The formula of Theorem 2 reduces for $n=3$ to

$$
\text { index }(L)=-\frac{1}{16 \pi i} \int_{S_{\infty}^{2}} \operatorname{tr}(U d U d U),
$$

where $U=|\Phi|^{-1} \Phi$. The index can be expressed simply in terms of the magnetic charge as follows. Let $\lambda_{A}(x), \psi_{A}(x)$ be the eigenvalues and eigenvectors of $\Phi(x)$ :

$$
\Phi(x) \psi_{A}(x)=\lambda_{A}(x) \psi_{A}(x)
$$

with $\Phi(x)$ given by (4.1), $\lambda_{A}(x)$ are just $-T+m,-T+m+1, \ldots, T+m$. (The index only depends on $m / \mu$, so we take $\mu=1$.) Now it is easy to verify the elementary formula

$$
\begin{aligned}
\partial_{j} U(x)= & \sum_{\substack{A, B \\
\lambda_{A}(x) \lambda_{B}(x)<0}}\left[\frac{2}{\lambda_{A}(x)-\lambda_{B}(x)} \operatorname{sign} \lambda_{B}(x)\right. \\
& \left.\cdot\left(\psi_{A}(x), \partial_{j} \Phi(x) \psi_{B}(x)\right)\right] \psi_{A}(x) \psi_{B}(x)^{\dagger} .
\end{aligned}
$$

Here $(\cdot, \cdot)$ denotes the inner product in the finite-dimensional space of the matrix $\Phi(x)$. We can now perform the matrix multiplications and the trace in (4.4) working in the basis $\psi_{A}(x) . \partial_{j} \Phi(x)$ is easy to express in this basis. At each point $x$ let $\phi^{a}(x), \phi_{1}^{a}(x), \phi_{2}^{a}(x)$ be an orthonormal set of 3 -vectors. The linear combinations $T_{0}(x)=T^{a} \phi^{a}(x), T_{i}(x)=T^{a} \phi_{i}^{a}(x), i=1,2$ satisfy relations similar to (4.2). Now since $\phi^{a}$ is a unit vector, we have

$$
\begin{aligned}
& \partial_{j} \phi^{a}=c_{1 j} \phi_{1}^{a}+c_{2 j} \phi_{2}^{a} \\
& \partial_{j} \Phi(x)=\partial_{j}\left(\mu \phi^{a} T^{a}+m\right)=c_{1 j} T_{1}+c_{2 j} T_{2} .
\end{aligned}
$$

The matrix elements of $T_{i}(x)$ in the basis $\psi_{A}(x)$ are well known. Let the index $A$ in $\psi_{A}(x)$ be just the corresponding eigenvalue of $\Phi(x): A=-T,-T+1, \ldots, T$. Then

$$
\begin{aligned}
& \left(\psi_{A}, T_{1} \psi_{B}\right)=\frac{1}{2} \delta_{A, B-1}[T(T+1)-A(A+1)]^{1 / 2}+\frac{1}{2} \delta_{A, B+1}[T(T+1)-A(A-1)]^{1 / 2} \\
& \left(\psi_{A}, T_{2} \psi_{B}\right)=\frac{1}{2 i} \delta_{A, B+1}[T(T+1)-A(A-1)]^{1 / 2} \\
& -\frac{1}{2 i} \delta_{A, B-1}[T(T+1)-A(A+1)]^{1 / 2} .
\end{aligned}
$$

Let $\{m\}$ denote the largest eigenvalue of $\phi^{a} T^{a}$ smaller than $m$, or, if there is no such eigenvalue, the smallest eigenvalue of $\phi^{a} T^{a}$ minus one. (Note that $m$ has to be different from all the eigenvalues of $\phi^{a} T^{a}$ for $L$ to be Fredholm.) Then the only terms that contribute in (4.5) are $(A, B)=(\{m\},\{m\}+1)$ or $(\{m\}+1,\{m\})$. With this 
information, a short calculation gives

$$
\operatorname{tr}(U d U d U)=2 i[T(T+1)-\{m\}(\{m\}+1)] c_{1 i} c_{2 j} d x^{i} d x^{j} .
$$

Noting that

$$
\varepsilon_{a b c} \phi^{a} d \phi^{b} d \phi^{c}=c_{1 i} c_{2 j} d x^{i} d x^{j}
$$

and recalling the formula (4.3) for the magnetic charge we get

$$
\operatorname{index}(L)=[T(T+1)-\{m\}(\{m\}+1)] \cdot N .
$$

For arbitrary $\mu$ in (4.1) $m$ should be replaced by $\frac{m}{\mu}$ in this formula. For $N=1$, two cases have been studied by explicit solution in the literature [4]. They correspond to $T=\frac{1}{2},\left\{\frac{m}{\mu}\right\}=-\frac{1}{2}$, with index 1 , and $T=1,\left\{\frac{m}{\mu}\right\}=0$, with index 2. Our general formula is in agreement with the explicit results.

Finally a remark is in order. No vanishing theorem is known to be valid for the systems under consideration in this Section, i.e. it is not known whether it is necessary that either $k_{+}$or $k_{\text {_ }}$ defined in Sect. I be zero. This is easily seen to be true in the case of Euclidean SU(2) pseudoparticle systems [1]. In the case of fermions in monopole fields it cannot be asserted whether $L^{\dagger} L$ or $L L^{\dagger}$ has no bound states at all [16], so there is no easy vanishing theorem for this case.

\section{Conclusion: Relation to Anomalies}

We summarize certain points made in the paper and indicate how our derivation of the index theorem is related to the theory of the anomalous divergence of the axial vector current [6]. The first equation in Proposition 1b) or the regulated version of it, as explained in the remark following the proof of the Proposition, is familiar to theoretical physicists. $J_{z}^{i}(x, x)$ is the vacuum expectation value of the axial vector current operator for a Euclidean field theory in an external field; $z$ is the mass of the quantum field. $B_{z}(x, x)$ represents the so-called naive divergence the direct quantum counterpart of the classical divergence of $J_{z}^{i}(x, x)$. Finally $A_{z}(x, x)$ is the anomalous term in the divergence - a purely quantum effect. Lemma 4 shows that this anomalous term is zero for operators of the form (3.1). Unlike the case of pseudoparticle configuration, it is now not the anomaly but rather the integral of the axial vector current that gives the index, as indicated by the second equation of Proposition 1b).

It is well-known in physics that the anomaly - the $A_{z}(x, x)$ - term is a result of renormalization - the residual term after the regularizing cutoffs are taken to infinity. On the other hand, the nonvanishing surface integrals that we have found can be thought of as a different kind of anomalous effect. This can be understood in terms of determinantal anomalies as follows.

We start with a theory for which one subtraction is sufficient to annihilate the $A_{z}(x, x)$ term in Proposition 1. [That $A_{z}(x, x)$ can be cancelled by a sufficiently large number of subtractions is apparent from the definition of $A_{z}(x, y)$ by (2.4).] This would be the case for the one-dimensional example (3.14). The global version 
of Proposition $1 \mathrm{~b}$ ), which we may now call the global or integrated axial vector current equation, becomes

$$
f(z)-f\left(z^{\prime}\right)=\sigma(z)-\sigma\left(z^{\prime}\right)
$$

where

$$
\sigma(z)=\int_{S_{\infty}^{n-1}} d S_{i} J_{z}^{i}(x, x)
$$

a surface term. In particular,

$$
f(z)-f(\infty)=\sigma(z)-\sigma(\infty) .
$$

The anomaly has been transferred to $f(\infty)$ and $\sigma(\infty)$. We emphasize that these quantities represent global anomalies in contrast to local ones, in the sense that it is not necessarily true that $\lim _{z \rightarrow \infty} \int B_{z}(x, x) d x=\int \lim _{z \rightarrow \infty} B_{z}(x, x) d x$.

Now consider the determinant

$$
D(z)=\operatorname{det}\left(L^{\dagger} L+z\right)\left(L L^{\dagger}+z\right)^{-1} .
$$

Formally, or else if $L$ is a finite-dimensional matrix, $D(z)=1, z \notin R^{-}$. Anomalies will modify this result. Now use the following formula for the regularized determinant of an operator $A$ :

$$
\ln \operatorname{det} A A_{0}^{-1}=\int_{0}^{\infty} \frac{d s}{s} \operatorname{Tr}\left(e^{-s A}-e^{-s A_{0}}\right)
$$

to obtain

$$
\begin{aligned}
\frac{d}{d z} \ln D(z) & =\int_{0}^{\infty} \frac{d s}{s} \frac{d}{d z} \operatorname{Tr}\left[e^{-s\left(L^{\dagger} L+z\right)}-e^{-s\left(L L^{\dagger}+z\right)}\right] \\
& =\operatorname{Tr}\left(\frac{1}{L^{\dagger} L+z}-\frac{1}{L L^{\dagger}+z}\right)=\frac{1}{z} f(z)
\end{aligned}
$$

provided the last trace exists, as it does in the case of the one-dimensional example (3.14). For more general cases the argument can probably be refined but we will not go into any details. Using (5.1) in (5.3) we obtain

$$
D(z)=D_{0}\left(\frac{z}{z_{0}}\right)^{f(\infty)} e_{z_{0}}^{\int_{0}^{z} d z^{\prime} \cdot z^{\prime}-1}\left[\sigma\left(z^{\prime}\right)-\sigma(\infty)\right] .
$$

Thus $f(\infty)$ accounts for an anomalous power behavior of $D(z)$ and $\sigma(z)-\sigma(\infty)$ for other variations from the constant value 1 .

We now show how $D(z)$ can be computed in essentially closed form. Specialize, as an illustration to (3.14), to avoid complicated questions. Further assume $A(x)$ is asymptotically unitary in (3.14). Using

$$
\lim _{\operatorname{Re} z \rightarrow \infty} \operatorname{Tr}\left(\frac{z}{L^{\dagger} L+z}-\frac{z}{L L^{\dagger}+z}\right)=0
$$


one can show that

$$
\lim _{t \rightarrow 0+}\left(e^{-t L^{\dagger} L}-e^{-t L L^{\dagger}}\right)=0
$$

and

$$
\lim _{z \rightarrow \infty} D(z)=1
$$

by making use of (5.2). Thus we find

$$
D(z)=e_{\infty}^{z} d z^{\prime} \cdot x^{\prime-1} \sigma\left(z^{\prime}\right)=\left(\frac{\sqrt{1+z}-1}{\sqrt{1+z}+1}\right)^{\operatorname{index}(L)},
$$

where $\sigma(z)$ is known by the results of Section 3:

$$
\sigma(z)=\frac{1}{(1+z)^{1 / 2}} \operatorname{index}(L) .
$$

Similar results are assumed to hold in higher dimensions. In writing (5.3) we have to face certain technicalities with the traces. $\sigma(z)$ is again expected to be possible to express in closed form. Guided by the example at the end of Section 3 we are led to conjecture

a) $\sigma(z)$ is invariant under $L$-compact perturbation.

b) $\sigma(z)$ is given by (3.3) if $\Phi(x)$ is only asymptotically unitary. [This is a consequence of a).]

Acknowledgements. I thank R. Jackiw for suggesting this problem and for various suggestions and discussions at the various stages of its solution. I would also like to express my warmest thanks to R. Bott, R. Seeley, and T. Spencer for interesting and helpful conversations.

\section{References}

1. For an introduction see Jackiw, R., Rebbi, C.: Phys. Rev. D16, 1052 (1977); Nielsen, N., Schroer, B. : Nucl. Phys. B127, 493 (1977); Ansourian, M.M.: Phys. Lett. 70B, 301 (1977)

2. Schwartz, A.: Phys. Lett. 67B, 172 (1977); Atiyah, M., Hitchin, N., Singer, I.: Proc. Nat. Acad. Sci., USA 74, 2662 (1977); Brown, L., Carlitz, R., Lee, C.: Preprint No. RL0-1388-724 University of Washington, 1977; Bernard, C., Christ, N., Guth, A., Weinberg, E.: Phys. Rev. D (in press); Bernard, C., Guth, A., Weinberg, E. : Preprint No. CU-TP-107, Columbia University 1977

3. Atiyah, M., Singer, I.: Ann. Math. 87, 484 (1968)

4. Jackiw, R., Rebbi, C.: Phys. Rev. D13, 3398 (1976)

5. Atiyah, M., Bott, R., Patodi, V.: Inventiones math. 19, 279 (1973); Gilkey, P.: The index theorem and the Heat equation. Boston: Publish or Perish 1974

6. Adler, S.: Perturbation Theory Anomalies. In: 1970 Brandeis University Summer Institute in Theoretical Physics (eds. S. Deser, M. Grisaru, H. Pendelton). Cambridge, Mass.: MIT Press 1970; Jackiw, R.: Field-Theoric Investigations. In: Lectures on current algebra and its applications. Princeton: Princeton University Press 1972

7. For basic definitions and general analytical theorems on Fredholm operators and the index, see, e.g., Goldberg, S. : Unbounded linear operators. New York : McGraw Hill 1966; or the long review article, Gokhberg, J.I., Krein, M. : Usp. Mat. Nauk 12, 43-118 (1957) translated in : Am. Math. Soc. Transl., Ser. 2, 13, 185 (1960)

8. Dunford, N., Schwartz, J.T.: Linear operators, Part II. New York: Interscience 1963

9. Seeley, R.T.: Brandeis University and Battelle Institute (1968) (unpublished); also: Preprint, University of Massachusetts (1978) 
10. See, for example, Seeley, R.T.: Trans. Am. Math. Soc. 117, 167-204 (1965), p. 173

11. This is essentially the Pauli-Villars regularization procedure that is well-known in field theory; see, e.g., Bjorken, J.D., Drell, S.D.: Relativistic quantum mechanics, p. 154. New York: McGraw Hill 1964

12. Lieb, E.H., Thirring, W.E.: In: Studies in mathematical physics, (eds. E.H. Lieb, B. Simon, A.S. Wightmann), p. 301. Princeton: Princeton University Press 1976

13. This index formula in one dimension can be derived as a corollary to the index theorem on compact manifolds with boundary proved in : Atiyah, M., Patodi, V., Singer, I. : Math. Proc. Camb. Phil. Soc. 77, 43 (1975). Here, however, we have given a direct open space proof that can be adapted to higher dimensions

14. 't Hooft, G.: Nucl. Phys. B79, 276 (1974); Polyakov, A. M.: Ah. Eksp. Teor. Fiz. Pis'ma Red. 20, 430 (1974) [JETP Lett. 20, 194 (1974)]

15. Arafune, J., Freund, P.G.O., Goebel, C.J.: J. Math. Phys. 16, 433 (1975)

16. Callias, C.J.: Phys. Rev. D16, 3068 (1977)

Communicated by A. Jaffe

Received December 21, 1977; in revised form April 10, 1978 